\title{
Distribution function in quantal cumulant dynamics
}

\author{
Yasuteru Shigeta ${ }^{\text {a) }}$ \\ Department of Physics, Graduate School of Pure and Applied Sciences, University of Tsukuba, \\ Tennodai 1-1-1, Ibaraki 305-8571, Japan
}

(Received 29 February 2008; accepted 8 April 2008; published online 30 April 2008)

\begin{abstract}
We have derived a quantum distribution function in terms of cumulants that are expectation values of a (anti)symmetric-ordered product of position and momentum fluctuation operators. A second-order approximation leads a Gaussian distribution function, which is positive definite and has proper marginals so that the Shannon entropy can be evaluated. (C) 2008 American Institute of Physics. [DOI: 10.1063/1.2917799]
\end{abstract}

In 1932, Wigner first opened a new field of quantum mechanics described in the phase space of position and momentum. He studied a function $F(q, p)=\int \psi^{*}(q+s / 2) \psi(q$ $-s / 2) \exp (-i p s) d s$, which yields the proper quantum mechanical marginal distribution function. ${ }^{1}$ This function has the remarkable property that it can be used to calculate a class of quantum mechanical averages in the same manner as the classical phase space distribution function is used to calculate classical averages. Therefore, it has been applied to a wide variety of fields such as quantum statistical mechanics, quantum optics, and questions relating to the foundations of a quantum-classical correspondence. ${ }^{2-4}$

As Wigner pointed out in his first paper, the function cannot be considered a proper probability distribution, because it may take on negative values. For this reason the function has a poor correspondence to the classical distribution function and is sometimes called a quasiprobability. The Husimi function is the simplest class of Gaussian smoothing of the Wigner distribution and leads to a non-negative distribution. ${ }^{5}$ Although the Husimi function reveals a better correspondence to the probability distribution function in classical mechanics than does the Wigner function, the former loses the property of the proper marginal distribution. There are a few distribution functions that fulfill both the non-negativity and proper marginal conditions as summarized in Table 2 of Ref. 4.

Prezhdo et al. developed the quantized Hamilton dynamics (QHD) approach by means of Heisenberg equations of motion (EOM) ${ }^{6-8}$ Recently we derived the coupled EOM of cumulants as an extension of the QHD method. ${ }^{9,10}$ We have called the method the quantal cumulant dynamics (QCD). In this Communication, the same scheme is applied to evaluate the quantum distribution function and clarify its properties in relation to physical meanings of cumulants.

First, we evaluate the density and the momentum density based on the QCD method. We use dimensionless units such that the Planck constant $\hbar=1$ and consider the usual Hermitian position $\hat{Q}$ and conjugate momentum $\hat{P}$ to be dimensionless operators, which obey the usual canonical commutation relations for a boson and the anticommutation relations for a

${ }^{a)}$ Electronic mail: shigeta@comas.frsc.tsukuba.ac.jp. fermion. In the QCD theory, the central variables are an expectation value of the position operator with respect to a given wave function $\psi, q=\langle\hat{Q}\rangle$, that of the momentum operator $p=\langle\hat{P}\rangle$, and cumulants ${ }^{11,12}$ of $n$ th- and $m$ th- order with respect to the position and momentum operators $\lambda_{n, m}$, where the bracket means $\langle\cdots\rangle=\langle\psi|\cdots| \psi\rangle$. Hereafter, we will refer $q$ and $p$ as the classical and $\lambda_{n, m}$ as the cumulant variables. For example, the lower-order cumulants are defined to be $\lambda_{2,0}$ $\equiv\left\langle{\hat{\lambda_{2,0}}}\right\rangle=\left\langle\delta \hat{Q}^{2}\right\rangle, \lambda_{1,1} \equiv\left\langle{\hat{\lambda_{1,1}}}\right\rangle\left\langle(\delta \hat{Q} \delta \hat{P})_{ \pm}\right\rangle$, and $\lambda_{0,2} \equiv\left\langle{\hat{\lambda_{0,2}}}\right\rangle$ $=\left\langle\delta \hat{P}^{2}\right\rangle$, where the subscript \pm denotes a symmetric-ordered product for the boson (upper sign) and an antisymmetricordered product for the fermion (lower sign), i.e., $(\hat{A} \hat{B})_{ \pm}$ $=1 / 2(\hat{A} \hat{B} \pm \hat{B} \hat{A})$, and $\delta \hat{A} \equiv \hat{A}-\langle\hat{A}\rangle$ is a fluctuation operator of $\hat{A}$.

The density is defined as the expectation value of the delta function, then we have

$$
\rho\left(q_{0}\right)=\left\langle\delta\left(\hat{Q}-q_{0}\right)\right\rangle=\left.\left\langle\exp \left[\delta \hat{Q} \frac{\partial}{\partial q_{1}}\right]\right\rangle \delta\left(q_{1}-q_{0}\right)\right|_{q_{1}=q}
$$

where we have used the shift operator representation. ${ }^{9}$ There seems a strange expression in Eq. (1), because the delta function exists without any integral. It should be stressed here that the expectation value of the shift operator is still a function of a differential operator. Since the differential form can be transformed into an integral form via the Fourier transformation, this expression is formally valid. By means of the density $\rho\left(q_{0}\right)$, the expectation value of an arbitrary analytic function $f(\hat{Q})$ can be written as

$$
\langle f(\hat{Q})\rangle=\int \rho\left(q_{0}\right) f\left(q_{0}\right) d q_{0} .
$$

By using the cumulant expansion techniques, ${ }^{9,11,12}$ the density can be expressed in terms of an infinite series of the cumulant variables as 


$$
\rho\left(q_{0}\right)=\left.\exp \left(\sum_{n=2}^{\infty} \frac{\lambda_{n, 0}}{n !} \frac{\partial^{n}}{\partial q_{1}^{n}}\right) \delta\left(q_{0}-q_{1}\right)\right|_{q_{1}=q}
$$

In the previous works, ${ }^{9,10}$ we have retained only secondorder cumulants. We assume that the density is approximated by using the second-order cumulants and the exponential function of the differential operator can be evaluated by the Fourier convolution techniques ${ }^{9}$ as

$$
\rho_{2}\left(q_{0}\right)=\left(2 \pi \lambda_{2,0}\right)^{-1 / 2} \exp \left(-\left(q_{0}-q\right)^{2} /\left(2 \lambda_{2,0}\right)\right)
$$

With the same manner, one can obtain an approximate momentum density as

$$
\pi_{2}\left(p_{0}\right)=\left(2 \pi \lambda_{0,2}\right)^{-1 / 2} \exp \left(-\left(p_{0}-p\right)^{2} /\left(2 \lambda_{0,2}\right)\right) .
$$

Both the second-order density and momentum density are described as the Gaussian distributions centered at corresponding classical variables and normalized to be unity.

Second, we proceed to evaluate the quantum distribution function (joint density) based on the cumulant expansion techniques. The joint density is defined as the expectation value of the (anti) symmetric-ordered product of two delta functions as follows:

$$
\rho_{ \pm}^{\text {joint }}\left(q_{0}, p_{0}\right)=\left\langle\hat{\rho}_{ \pm}^{\text {joint }}\left(q_{0}, p_{0}\right)\right) \equiv\left\langle\left[\delta\left(\hat{Q}-q_{0}\right) \delta\left(\hat{P}-p_{0}\right)\right]_{ \pm}\right\rangle .
$$

The expectation value of an arbitrary analytic function that consists of (anti)symmetric-ordered products of the position and momentum operators $f_{ \pm}(\hat{Q}, \hat{P})$ is written in terms of the joint density as

$$
\begin{aligned}
\left\langle f_{ \pm}(\hat{Q}, \hat{P})\right\rangle & =\iint \rho_{ \pm}^{\text {joint }}\left(q_{0}, p_{0}\right) f\left(q_{0}, p_{0}\right) d q_{0} d p_{0} \\
& =I\left(f\left(q_{0}, p_{0}\right)\right),
\end{aligned}
$$

where the corresponding $c$-number analytic function $f\left(q_{0}, p_{0}\right)$ can be simply obtained by neglecting the (anti)symmetric order. We also applied generalized cumulant expansion techniques to the joint density, and then we have

$$
\begin{aligned}
\rho_{ \pm}^{\text {joint }}\left(q_{0}, p_{0}\right)= & \exp \left[\sum_{m+n=2}^{\infty} \frac{\lambda_{m, n}}{m ! n !} \frac{\partial^{m+n}}{\partial q_{1}^{m} \partial p_{1}^{n}}\right] \delta\left(q_{1}-p_{0}\right) \\
& \times\left.\delta\left(p_{1}-p_{0}\right)\right|_{q_{1}=q, p_{1}=p} .
\end{aligned}
$$

Using the second-order cumulants and the classical variables, the second-order joint density can be evaluated to be

$$
\rho_{2, \pm}^{\text {joint }}\left(q_{0}, p_{0}\right)=\frac{\gamma^{-1 / 2}}{2 \pi} \exp \left[\frac{-\lambda_{0,2}\left(q-q_{0}\right)^{2}+2 \lambda_{1,1}\left(q-q_{0}\right)\left(p-p_{0}\right)-\lambda_{2,0}\left(p-p_{0}\right)^{2}}{2 \gamma}\right]
$$

where $\gamma=\lambda_{2,0} \lambda_{0,2}-\lambda_{1,1}^{2}$. Since the expectation value of the symmetric-ordered product is real, $\gamma$ must be positive, i.e., $\lambda_{2,0} \lambda_{0,2}>\lambda_{1,1}^{2}$. Note that this joint density is normalized to be unity, i.e., $I(1)=\iint \rho_{2}^{\text {joint }}\left(q_{0}, p_{0}\right) d q_{0} d p_{0}=1$. Since all the cumulant and classical variables are real, the joint density is positive-definite. It is easily shown that this joint density has proper marginals

$$
\begin{aligned}
& \rho_{2}\left(q_{0}\right)=\int \rho_{2, \pm}^{\text {joint }}\left(q_{0}, p_{0}\right) d p_{0} \\
& \pi_{2}\left(p_{0}\right)=\int \rho_{2, \pm}^{\text {joint }}\left(q_{0}, p_{0}\right) d q_{0} .
\end{aligned}
$$

Furthermore, the joint density has the following boundary conditions:

$$
\begin{aligned}
& I\left(q_{0}\right)=q, \\
& I\left(p_{0}\right)=p, \\
& I\left(\left(q-q_{0}\right)^{2}\right)=\lambda_{2,0}, \\
& I\left(\left(p-p_{0}\right)^{2}\right)=\lambda_{0,2},
\end{aligned}
$$

$$
I\left(\left(q-q_{0}\right)\left(p-p_{0}\right)\right)=\lambda_{1,1}
$$

By means of these five variables, one can evaluate any expectation value under the second-order cumulant approximation. The Hamiltonian of a one-dimensional system is given by

$$
H(\hat{Q}, \hat{P})=\frac{1}{2} \hat{P}^{2}+V(\hat{Q})
$$

where the first and the second terms denote the kinetic and the potential energy operator, respectively. The second-order total energy is evaluated as

$$
E_{2}=I\left(H\left(q_{0}, p_{0}\right)\right)=\frac{1}{2}\left(p^{2}+\lambda_{0,2}\right)+\tilde{V}\left(q, \lambda_{2,0}\right),
$$

where we have used the abbreviation

$$
\tilde{V}\left(q, \lambda_{2,0}\right)=\int \rho_{2}\left(q_{0}\right) V\left(q_{0}\right) d q_{0}
$$

for simplicity. To derive the expression, we have used the proper marginal conditions. Note that the total energy is independent of $\lambda_{1,1}$.

In order to recognize the role of the cumulant variables, we depict a unit ellipse of the exponent of Eq. (9), 


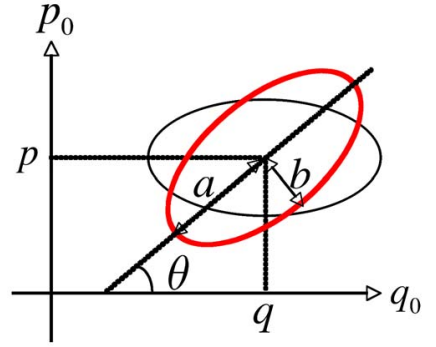

FIG. 1. (Color online) A unit ellipse of exponent in second-order distribution function centered at $\left(q_{0}, p_{0}\right)$ for $\lambda_{1,1} \neq 0$ (red) and $\lambda_{1,1}=0$ (black).

$$
1=\frac{1}{2 \gamma}\left(\left(q-q_{0}\right)\left(p-p_{0}\right)\right)\left(\begin{array}{cc}
\lambda_{0,2} & -\lambda_{1,1} \\
-\lambda_{1,1} & \lambda_{0,2}
\end{array}\right)\left(\begin{array}{c}
\left(q-q_{0}\right) \\
\left(p-p_{0}\right)
\end{array}\right)
$$

in Fig. 1. Introducing a rotation matrix that diagonalizes the above matrix, we have a rotation angle $\theta$, major semiaxis $a$, and minor semiaxis $b$ as follows:

$$
\begin{aligned}
& \theta=\frac{1}{2} \tan ^{-1}\left[2 \lambda_{1,1} /\left(\lambda_{2,0}-\lambda_{0,2}\right)\right], \\
& a=\max \left(\frac{1}{2} \sqrt{\left(\lambda_{2,0}+\lambda_{0,2}\right) \mp \sqrt{\left(\lambda_{0,2}-\lambda_{2,0}\right)^{2}+4 \lambda_{1,1}^{2}}},,\right. \\
& b=\min \left(\frac{1}{2} \sqrt{\left(\lambda_{2,0}+\lambda_{0,2}\right) \mp \sqrt{\left(\lambda_{0,2}-\lambda_{2,0}\right)^{2}+4 \lambda_{1,1}^{2}}}\right) .
\end{aligned}
$$

Due to the Heisenberg's uncertainty relation, the expectation value of $(\delta \hat{Q} \delta \hat{P})_{ \pm}^{2}$ becomes

$$
\lambda_{2,0} \lambda_{0,2}+\lambda_{1,1}^{2} \geqslant \frac{1}{4} \text {. }
$$

Roughly speaking, this relation implies that $\lambda_{2,0}$ is inversely proportional to $\lambda_{0,2}$. If we assume the minimal uncertainty relation, i.e., $\lambda_{2,0} \lambda_{0,2}+\lambda_{1,1}^{2}=\frac{1}{4}$, and $\lambda_{1,1}=0$, the density is identical to that obtained in terms of a squeezed coherent state. Thus, one can yield position and momentum squeezed states by adjusting $\lambda_{2,0}$ and $\lambda_{0,2}$. When $\lambda_{1,1}=0$, the angle is zero; i.e., the ellipse does not rotate with respect to the $q-p$ axis. The major and minor semiaxes depend on all cumulant variables. In particular, as $\lambda_{1,1}$ and the difference between $\lambda_{2,0}$ and $\lambda_{0,2}$ increase, the difference between the major and minor semiaxes becomes larger.

Judging from the resultant density, the second-order cumulant method is almost equivalent to several other methods, such as the Gaussian wave packet method of Heller, ${ }^{13,14}$ the Jackiw-Kerman approach, ${ }^{15}$ and the squeezed coherent state approach of Tsue and Fujiwara, ${ }^{16}$ when we assume the minimal uncertainty condition. The number of independent variables in those approaches for the one-dimensional problem is 4 , while it is 5 in this approach. In particular, two of the three cumulants have a clear physical meaning. $\lambda_{2,0}$ and $\lambda_{0,2}$ are related to the width of the Gaussian wave packet in the position and the momentum space, respectively. We demonstrated that $\lambda_{1,1}$ contributes both to the rotation with respect to the $q-p$ plane and the squeezing of the distribution.

Third, we consider a Liouville equation of the joint density operator up to the second order. Heisenberg EOM for the expectation value of the joint density operator is given by

$$
\dot{\rho}_{ \pm}^{\text {joint }}\left(q_{0}, p_{0} ; t\right)=-i\left\langle\left[\hat{\rho}_{ \pm}\left(q_{0}, p_{0} ; t\right), \hat{H}\right]_{-}\right\rangle,
$$

where $[\cdots, \cdots]_{-}$is a commutator and $\hat{A}(t)$ $=\exp [i \hat{H} t] \hat{A} \exp [-i \hat{H} t]$ is operator $\hat{A}$ in the Heisenberg representation. Since the joint density depends only on the classical and cumulant variables, in terms of the chain rule, we have

$$
\dot{\rho}^{\text {joint }}\left(q_{0}, p_{0} ; t\right)_{ \pm}=\sum_{X} \dot{X}(t) \frac{\partial \rho^{\text {joint }}\left(q_{0}, p_{0} ; t\right)}{\partial X(t)},
$$

where $X=q, p$, and $\lambda_{n, m}(m+n=2, \ldots, \infty)$. Within the secondorder approximation, substituting the EOMs of classical and second-order cumulant variables ${ }^{9}$ in Eq. (26) leads to

$$
\begin{aligned}
\dot{\rho}_{2, \pm}^{\text {joint }}\left(q_{0}, p_{0} ; t\right)= & \left(\left(q(t)-q_{0}\right) \lambda_{1,1}(t)-\left(p(t)-p_{0}\right) \lambda_{2,0}(t)\right) \\
& \times\left(\left(q(t)-q_{0}\right) \tilde{V}^{(2)}(t)-\widetilde{V}^{(1)}(t)\right) \\
& \times \rho_{2, \pm}^{\text {joint }}\left(q_{0}, p_{0} ; t\right),
\end{aligned}
$$

where $\widetilde{V}^{(n)}(t)$ is the $n$th derivative of $\tilde{V}\left(q(t), \lambda_{2,0}(t)\right)$ with respect to $q(t)$. This EOM should be solved with the EOMs of the classical and cumulant variables. Initial conditions are obtained from the least quantum energy principle initially suggested by Tsue and Fujiwara, which gives the exact ground state energy and density for a harmonic oscillator.

Finally, we consider an entropy of the distribution function. Since the joint density devised here is positive definite, one can define the entropy by means of the joint density. The Shannon entropy is given by

$$
\begin{aligned}
S_{2}^{\text {joint }}(t) & =-\iint \rho_{2, \pm}^{\text {joint }}\left(q_{0}, p_{0} ; t\right) \ln \rho_{2, \pm}^{\text {joint }}\left(q_{0}, p_{0} ; t\right) d q_{0} d p_{0} \\
& =2.837877+\frac{1}{2} \ln \gamma(t) .
\end{aligned}
$$

The Shannon entropy is independent of time, because the derivative of $\gamma(t)$ with respect to time is identically zero. We define here a Lagrangian that is a sum of the Shannon entropy and the boundary conditions with Lagrange multipliers $\tau_{x}$ as

$$
L_{2}=S_{2}^{\mathrm{joint}}(t)+\sum_{X} \tau_{X}\left[I\left(X\left(q_{0}, p_{0}\right)\right)-\langle\hat{X}\rangle\right],
$$

where $X=\left(q, p, \lambda_{2,0}, \lambda_{1,1}\right.$, and $\left.\lambda_{0,2}\right)$ and $X\left(q_{0}, p_{0}\right)$ is the corresponding $c$-number function. It is easy to prove that the entropy $S_{2}$ is maximized subject to the constraints that the joint density leads the expectation values given in Eqs. (12)-(16), since $d L_{2} / d \rho_{2, \pm}^{\mathrm{joint}}=0$ and $d^{2} L_{2} / d\left(\rho_{2, \pm}^{\mathrm{j} \text { jint }}\right)^{2}<0$. These relations were previously demonstrated by Rajagopal as an extension of the classical probability function with constraints on the quantum expectation values based on the maximum entropy method. ${ }^{17}$ Thus, the joint density is a variational solution that maximizes the Shannon entropy.

In this Communication, we restrict ourselves to treating second-order cumulant variables for the one-dimensional cases to show the basic properties that the distribution function in QCD exhibit. Of course, the method is applicable to many-particle multi-dimensional cases. In particular, we performed the second-order QHD simulation of fourth-order 
multidimensional $a b$ initio vibronic Hamiltonians ${ }^{8}$ and found that the obtained vibrational frequencies are in good accordance with those obtained by the vibrational configuration interaction method. Since the QCD with the truncated potential is equivalent to the QHD, both QHD and QCD are powerful tools for simulating the molecular vibrations. Since each vibrational mode is expressed as a different boson, the total density is described as a product of densities of all vibrational degrees of freedom. The other issues are how to include higher-order cumulant variables, which leads to nonGaussian distributions, and how to take its statistics into account. These extensions will be done in future work.

\footnotetext{
${ }^{1}$ E. Wigner, Phys. Rev. 40, 749 (1932).

${ }^{2}$ M. Hillery, R. F. O'Connell, M. O. Scully, and E. P. Wigner, Phys. Rep. 106, 121 (1985).

${ }^{3}$ K. Takahashi, Prog. Theor. Phys. Suppl. 98, 109 (1989).
}

${ }^{4}$ H.-W. Lee, Phys. Rep. 259, 147 (1995).

${ }^{5}$ K. Husimi, Proc. Phys. Math. Soc. Jpn. 22, 264 (1940).

${ }^{6}$ O. V. Prezhdo and Y. V. Pereverzev, J. Chem. Phys. 113, 6557 (2000); 116, 4450 (2002).

${ }^{7}$ O. V. Prezhdo, Theor. Chem. Acc. 116, 206 (2006), and references cited therein.

${ }^{8}$ H. Miyachi, Y. Shigeta, and K. Hirao, Chem. Phys. Lett. 432, 585 (2006).

${ }^{9}$ Y. Shigeta, H. Miyachi, and K. Hirao, J. Chem. Phys. 125, 244102 (2006).

${ }^{10}$ Y. Shigeta, H. Miyachi, and K. Hirao, Chem. Phys. Lett. 443, 414 (2007).

${ }^{11}$ J. E. Mayer, J. Chem. Phys. 5, 67 (1937).

${ }^{12}$ R. Kubo, J. Phys. Soc. Jpn. 17, 1100 (1962).

${ }^{13}$ E. J. Heller, J. Chem. Phys. 62, 1544 (1975); 64, 63 (1976).

${ }^{14}$ A. K. Pattanayak and W. C. Schieve, Phys. Rev. E 50, 3601 (1994).

${ }^{15}$ R. Jackiw and A. Kerman, Phys. Lett. 71, 158 (1979).

${ }^{16}$ Y. Tsue and Y. Fujiwara, Prog. Theor. Phys. 86, 443 (1991).

${ }^{17}$ A. K. Rajagopal, Phys. Rev. A 27, 558 (1983). 